\title{
Artificial Opals as Nanophotonic Materials for Optical Communication
}

Lavrinenko, Andrei; Leyrer, Reinhold J.; Wohlleben, Wendel; Dissaux, Nolwenn; Heggarty, Kevin; Boyle, Mark; Kiyan, Roman; Neumeister, Andre

\section{Published in:}

Proceedings of $9<$ sup $>$ th $</$ sup $>$ International Conference on Transparent Optical Networks

Link to article, DOI:

10.1109/ICTON.2007.4296182

Publication date:

2007

Document Version

Publisher's PDF, also known as Version of record

Link back to DTU Orbit

Citation $(A P A)$ :

Lavrinenko, A., Leyrer, R. J., Wohlleben, W., Dissaux, N., Heggarty, K., Boyle, M., Kiyan, R., \& Newmeister, A. (2007). Artificial Opals as Nanophotonic Materials for Optical Communication. In Proceedings of 9 International Conference on Transparent Optical Networks (Vol. 2, pp. 208-211). IEEE.

https://doi.org/10.1109/ICTON.2007.4296182

\section{General rights}

Copyright and moral rights for the publications made accessible in the public portal are retained by the authors and/or other copyright owners and it is a condition of accessing publications that users recognise and abide by the legal requirements associated with these rights.

- Users may download and print one copy of any publication from the public portal for the purpose of private study or research.

- You may not further distribute the material or use it for any profit-making activity or commercial gain

- You may freely distribute the URL identifying the publication in the public portal 


\title{
Artificial Opals as Nanophotonic Materials for Optical Communication
}

\author{
Andrei V. Lavrinenko ${ }^{1}$, Reinhold J. Leyrer ${ }^{2}$, Wendel Wohlleben ${ }^{2}$, Nolwenn Dissaux ${ }^{3}$, Kevin Heggarty ${ }^{3}$ \\ Mark Boyle ${ }^{4}$, Roman Kiyan ${ }^{4}$, Andre Neumeister ${ }^{4}$ \\ ${ }^{1} \mathrm{COM} \cdot D T U$, Department of Communications, Optics \& Materials, NanoDTU, Technical University of \\ Denmark, 345v, DK-2800, Kgs. Lyngby, Denmark \\ ${ }^{2}$ Polymer Research, BASF Aktiengesellschaft, 67056 Ludwigshafen, Germany \\ ${ }^{3}$ GET-ENST Bretagne, Département d'Optique, 29238 Brest, France \\ ${ }^{4}$ Laser Zentrum Hannover e.V., Hollerithallee 8, 30419 Hannover, Germany \\ Tel: (45) 4525 6392, Fax: (45) 4593 6581, e-mail: ala@com.dtu.dk
}

\begin{abstract}
We report on advances in the fabrication of thick and homogeneous polymer opals films with bead sizes up to $600 \mathrm{~nm}$; photo-ablation and point and line defect inscription with femtosecond laser pulses; analysis of the influence of imperfections on opal transmission-reflection properties and numerical and experimental characterization of fabricated samples.

Keywords: three-dimensional photonic crystals, two-photon polymerization, vertical deposition, laser diffraction, imperfections.
\end{abstract}

\section{INTRODUCTION}

It is well known that only 3-dimensional photonic crystals can provide a complete photonic band gap. There is a strong motion to utilize such structures as templates for further manufacturing of on-chip photonic circuits. Our strategy towards implementation of artificial opals as novel photonic materials suitable for optical communications in the visible and near infrared ranges comprises research activities in: (i) fabrication of polymer based millimetre-scale colloidal opals; (ii) laser based 3D defect inscribing with nm-resolution; (iii) infiltration and inversion techniques to realise complete photonic band gaps; (iv) simulation and design tools; (v) characterisation and performance evaluation of the manufactured components. Our latest achievements are presented in the relevant sections.

\section{INFLUENCE OF IMPERFECTIONS ON THE COMPLETE BANGGAP IN SI INVERSE OPLAS}

The quantitative study of disorder influence on light transmission in three-dimensional photonic crystals having a complete photonic band gap like Si-inverted opals is of great importance. It will settle the tolerance limits for fabrication processes. Disorder is implied as fluctuation in sizes and/or sites of air spheres. The straight transmission in the $\Gamma \mathrm{L}$ (111) direction through an 18-layers thick inverted opal cell is simulated by the 3D FDTD method. Two kinds of disorder distributions are used in modelling: Gaussian and a skew one obtained from checking the colloidal particles distribution before the segmentation into the polystyrene opal template. Transmission in the perfect FCC structure (deep $50 \mathrm{~dB}$ gap in the range $0.78-0.83$ ) is shown by the solid line in Fig. 1, left.
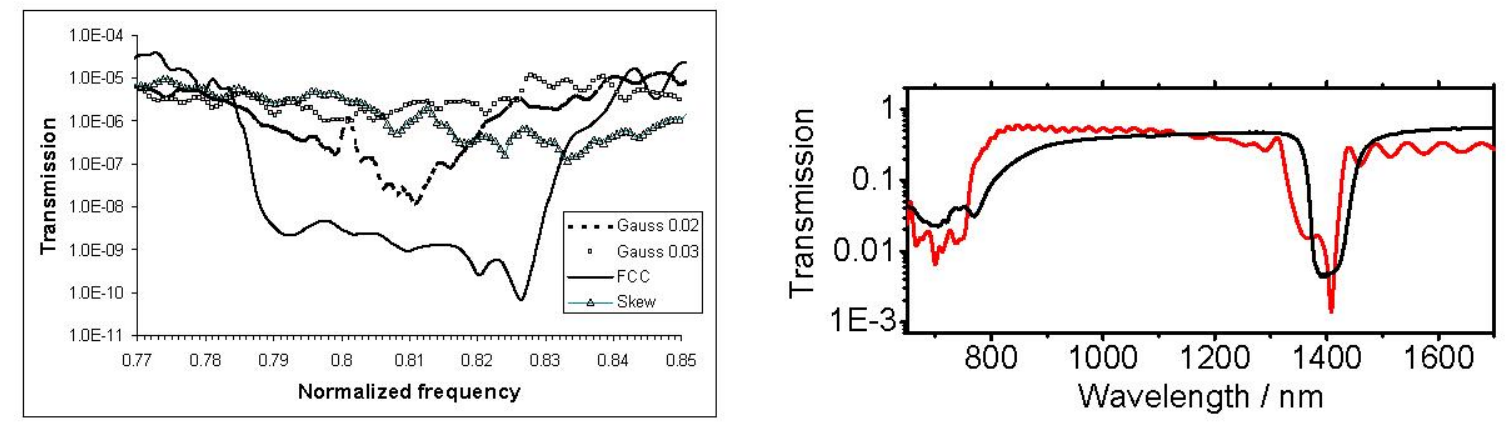

Figure 1. Left: Transmission spectra of a perfect Si-inverted opal film in the range of the complete bandgap and opal films with various embedded imperfections. Right: Calculated (red) and measured (black) transmission of reproduction $B$ sample (see Section 4 for details).

For finite structures the fragility of complete band gaps is worse than predicted for infinite crystals [1]. Our simulations show that band gap properties in transmission attenuation are severely distorted even with $2 \%$ disorder distributed by the Gaussian law (Gauss 0.02 in Fig. 3, left), while 3\% disorder closes the gap

This work is supported by the European Commission FP6, project NewTon, NMP4-CT-2005-017160. 
completely (Gauss 0.02 in Fig. 3, left). The skew distribution (Skew in Fig. 3, left), which can fit more or less to the $2 \%$ Gaussian, is likely to be the worst case. This postulates the tolerance limits for technology required to keep band gap open, pretending finite 3D photonic crystals to be a template for integrated circuits.

\section{FABRICATION OF POLYMER BASED COLLOIDAL CRYSTALS}

Emulsifier free emulsion polymerisation is one of the standard techniques to synthesize monodisperse polymer colloids (for an overview compare [2]). All polymer dispersions used for the crystals in this contribution were reproductions of the emulsifier free polymerisation with pre-polymerisation step [3]. Analytical Ultracentrifugation (AUC) is a fractionating colloid characterisation such that - in contrast to light scattering $-\mathrm{a}$ distribution of sizes is determined with high resolution. Furthermore - in contrast to TEM/SEM - the AUC integrates over $10^{9}-10^{12}$ particles, such that statistical relevance even of minor fractions is high [4]. It is evident that the actual high-resolution, high-statistics size distribution of polymer dispersions is not symmetrical but skewed with more coarse than fine fraction (Fig. 2a). Mean standard deviation is hence not an optimal measure of polydispersity, but for comparison, all present samples achieve a PI $=\left(D_{90}-D_{10}\right) / D_{50}=0.08-0.09$, and a mean standard deviation of $<2 \%$ from average particle diameter.

For self-assembly the polymer dispersions were diluted as is, without any purification step. We grow the photonic crystal in near-vertical deposition without any mechanical movement in order to fabricate large-volume thick crystals $[5,6]$. The contraction of the crystal in the latest stages of drying induces mechanical stress that ultimately leads to adhesive failure at the polymer-substrate interface. The stress limits the thickness of practical polymer opal templates in our case to $50-100 \mu \mathrm{m}$. All following results are obtained with $30 \pm 3 \mu \mathrm{m}$ thick crystals, corresponding to 60 layers.

\section{CHARACTERIZATION OF OPALS SAMPLES}

Crystalline arrangement can be verified either by microscopy approaches that record the position of each individual point of the lattice (such as scanning electron microscopes - SEM, atomic force microscopes - AFM, or optical), or by integrative methods that record cooperative effects (such as diffraction, reflection, transmission). However, little has been reported about the angular orientation of the (111) layer with respect to the substrate. This angular orientation of the symmetry axes within the (111) plane is evident from laser diffraction, and obviously, for our samples the line that is defined by the top two diffraction spots is parallel to the meniscus in the vertical deposition process. We reproduced the polymer dispersion in independent experiments, each followed by self-assembly, and then recorded laser diffraction patterns (Fig. 2c) [3]. The diffraction pattern shrinks with increasing particle sizes $\left(\mathrm{D}_{50}=583-597-624 \mathrm{~nm}\right.$ for reproductions $\left.\mathrm{A}-\mathrm{B}-\mathrm{C}\right)$ but the orientation is the same. Every sample has identical angular orientation, with the top two diffraction spots aligned with the meniscus (Fig. 2c).

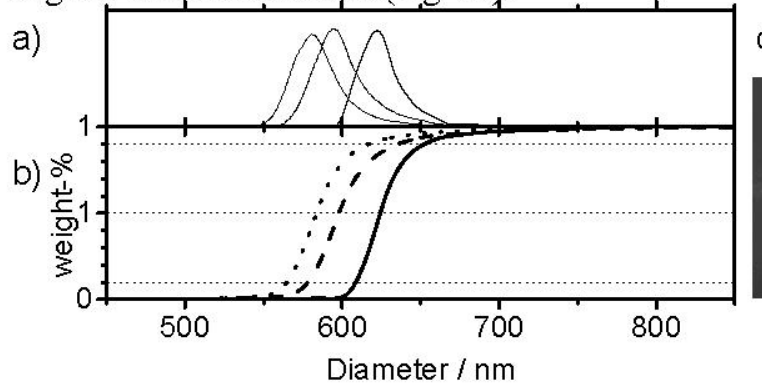

c)

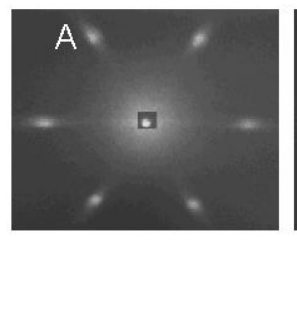

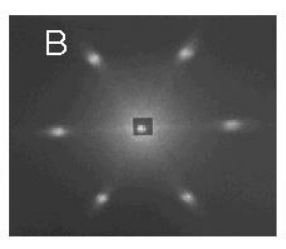

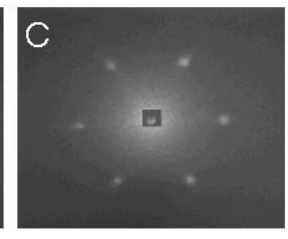

Figure 2. Complete reproductions of polymer synthesis and self-assembly. a) Differential and b) integral size distribution of reproductions $A$ dot), $B$ (dash), $C$ (solid) from $A U C$, integrating over $\sim 10^{9}$ particles in the original diluted suspension. The actual distribution of polymer dispersions is not symmetrical but skewed with more coarse than fine fraction. c) Laser diffraction of reproductions $A, B, C$ (beam diameter $6 \mathrm{~mm}$, the rectangular shadow is a beam-stop). The diffraction pattern shrinks due to the increasing particle size but the orientation of the diffraction pattern is identical, hence the angular orientation is pre-determined for all reproductions.

The opals fabricated using the techniques described above are characterized both structurally using Interferometric Optical Microscopes, AFM and SEM and for their optical properties via the study of the crystal diffraction patterns for different optical wavelengths and their transmission and reflection spectra across the visible and near Infra Red (IR) up to and beyond the targeted optical telecommunications wavelength bands around $1300 \mathrm{~nm}$ and $1550 \mathrm{~nm}$. Figure 3, left shows a SEM view confirming the hexagonal packing arrangement and allowing measurement of the crystal cell pitch to calibrate computer simulations. Fig. 3 , right is an interferometric microscope view on which grain size and layer step orientation can be used, with diffraction experiments (see Fig. 2c) to confirm the extent and orientation of macroscopic crystal order. 

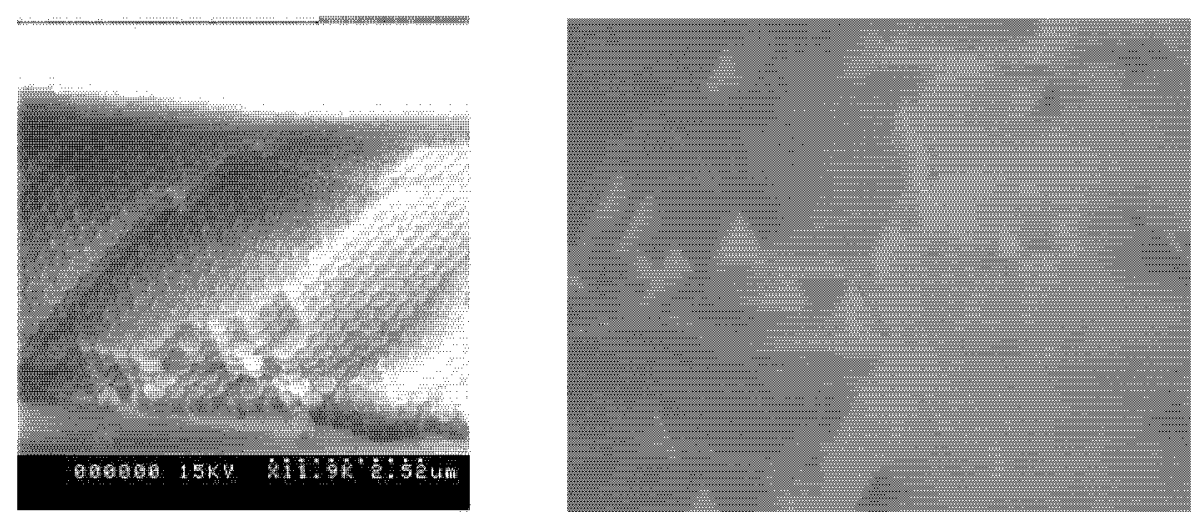

Figure 3. Left: SEM view of opal structure. Right: Interferometric microscope view of opal surface.

In our experimental setup used to measure the transmission and (with a slight modification) reflection spectra of the opals microscope objectives are used to illuminate small areas of the samples. The measurement of spectra from 400 to $1700 \mathrm{~nm}$ requires the use of two different photodetectors and filters to remove the higher diffraction orders of the monochromator grating. All spectra are calibrated by comparing spectra with and without the opal sample. The experimental transmission spectrum shown in Fig. 1, right shows a well defined photonic crystal stop band whose position and width are in close agreement with the 3D FDTD computer simulations (Fig. 1, right, red curve) and structural measurements.

\section{LASER BASED 3D DEFECT INSCRIBING}

Two methods have been used for this paper to create defects in a colloidal crystal: femtosecond micromachining and two photon polymerization (2PP). Femtosecond laser micromachining has been applied to structurally modify the surface and the material in the bulk in various types of materials, such as ceramics, metals, dielectrics, and polymers (see [7] and references therein). Due to the very short pulse duration of femtosecond pulses, the input energy cannot couple into the lattice, thus providing a method for melt-free structures and can achieve submicron dimensions. Another advantageous characteristic is the fact that the peak intensities associated with the ultrafast pulses allow multi-photon processes to occur. This feature is exploited in the $2 \mathrm{PP}$ technique $[8,9]$, where a photo-resist is polymerized with the simultaneous absorption of two infrared photons.

\subsection{Femtosecond Laser Micromachining}

Femtosecond laser micromachining has been used to create melt-free point defects and line defects on the surface of the colloidal crystals, as can be seen in Fig. 4. A single femtosecond laser pulse emitted from an amplified 80-fs Ti:Sapphire Laser system at $800 \mathrm{~nm}$ has been focused through a microscope objective $(\mathrm{NA}=0.95$ ) onto the surface of the colloid crystal. At an energy of 12nJ, a single layer of spheres has been removed (Fig. 4, left). The $1.1 \mu \mathrm{m}$ diameter is close to the theoretical diffraction free focal spot size. This "digital etching" has been observed previously with a nanosecond $\mathrm{N}_{2}$ laser [10].
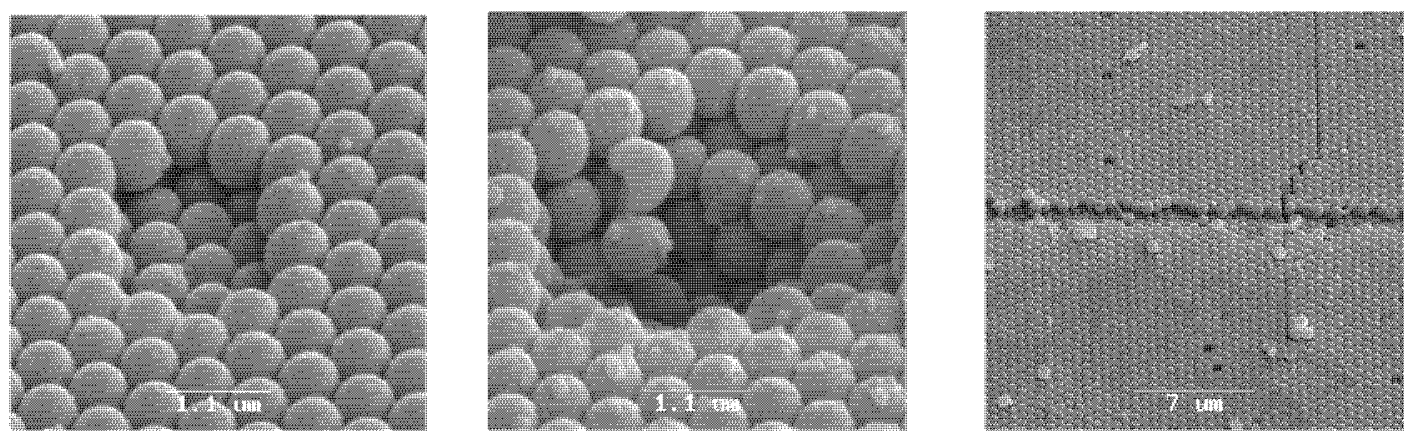

Figure 4. Left: Point defect on the surface of a colloid crystal with diameter 1.1 $\mu \mathrm{m}$. Center: a linear defect created with 50 pulses incident on the same point. Right: A linear defect on the surface has been achieved by scanning the colloid crystal with respect to the laser beam. The scanning speed was $100 \mu \mathrm{m} / \mathrm{s}$ and energy of $25 \mathrm{~nJ} / \mathrm{pulse}$ was used. The width of the linear defect is $1.5 \mu \mathrm{m}$.

Multiple pulses incident onto the same spot can remove successive layers, as shown in Fig. 4, effectively inscribing a vertical linear defect. In the midst of Fig. 4, a linear defect was inscribed 50 laser pulses of the same parameters. The depth is limited due to the strong focusing conditions of the microscope objective. By moving the colloid crystal with respect to the laser beam, a line defect can be inscribed on the surface of the colloid 
crystal as shown in Fig. 4 right. The colloid crystal was moved at a speed of $100 \mu \mathrm{m} / \mathrm{s}$ and the irradiation energy was $25 \mathrm{~nJ} /$ pulse.

\subsection{Two photon polymerization}

Defect inscription through two photon polymerization $[11,12]$ involves the infiltration of a photosensitive resin in the interstitial spaces between the spheres. This has been achieved with ORMORCER®) b59 (photo-initiator Igracure). The polymerization is achieved with a focussed femtosecond Ti:Sapphire laser oscillator at $800 \mathrm{~nm}$. The polymerization process results in a change in refractive index and thus can be observed in an optical microscope. The two-photon process limits the affected region to the focus, allowing for a true 3D structuring. In Fig. 5, linear defects are shown at different depths within the infiltrated colloid crystal. In these two optical microscopy pictures, four lines are visible (start indicated by the red arrows) in the image. The image on the left was written at a depth of $5 \mu \mathrm{m}$ below the surface, and the right image was realized at a depth of $7 \mu \mathrm{m}$. A resolution of $800 \mathrm{~nm}$ was achieved at a depth of $5 \mu \mathrm{m}$.
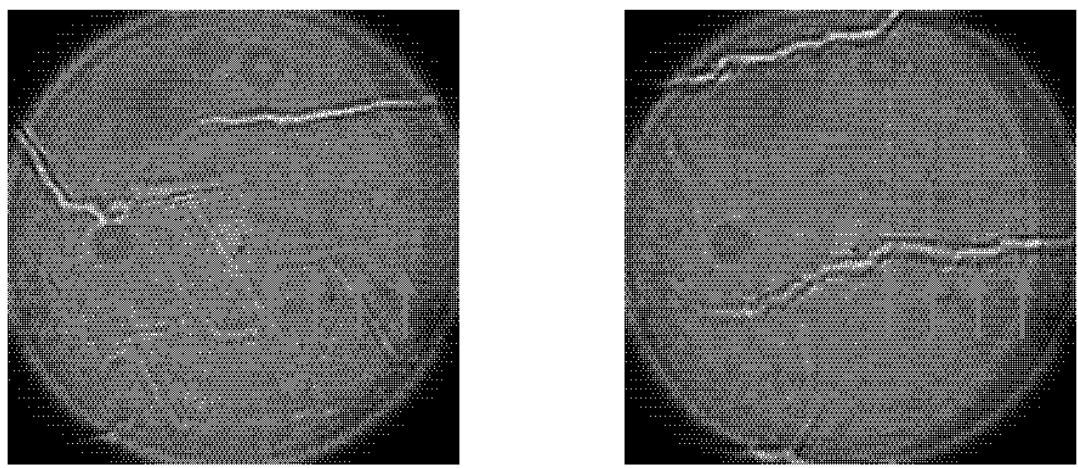

Figure 5. Optical microscopy images of the line defects inscribed into opal template infiltrated with ORMOCER by $2 P P$ process. The line defects have been inscribed at the distance $5 \mu m$ (left image) and $7 \mu m$ (right image) from the substrate/opal interface. Distance between lines is $5 \mu \mathrm{m}$. Line defects start at the tips of the arrows.

\section{CONCLUSIONS}

We have shown recent advances in different fabrication and modelling steps towards all-optical integrated circuits on the base of inverted opals. The polystyrene template has been proven to have high quality, and we demonstrated that various defects in 3D can be incorporated in the template with adequate quality too. To reach the final goal a reliable and accurate opal inversion process by high-index material infiltration is now required.

\section{REFERENCES}

[1] Z.-Y. Li, Z.-Q. Zhang: Fragility of photonic band gaps in inverse-opal photonic crystals, Phys. Rev. B, vol. 62 , pp.1516-1519, 2000.

[2] C.E. Reese, S.A. Asher: Emulsifier-free emulsion polymerization produces highly charged, monodisperse particles for near infrared photonic crystals, J. Colloid Interface Sci., vol. 248, pp. 41-46, 2002.

[3] W. Wohlleben, et al.: Large-volume polymer 3-D photonic crystals with pre-determined orientation and their full-crystal diffraction, $A d v$. Mater., submitted.

[4] H. Cölfen: Analytical Ultracentrifugation of nanoparticles, in Encyclopedia of Nanoscience and Nanotechnology, American Scientific Publishers, 2004, pp. 67-88.

[5] W. Wohlleben, et al.: Mechano-optical octave-tunable elastic colloidal crystals made from core-shell polymer beads with self-assembly techniques, Langmuir, vol. 23, pp. 2961-2969, 2007.

[6] D. J. Norris, et al.: Opaline photonic crystals: how does self-assembly work?, Adv. Mater., vol. 16, pp. 1393-1399, 2004.

[7] Loren Rachel Cerami, et al: : Femtosecond laser micromachining, in Ultrafast Optics, Rick Trebino and Jeff Squier Ed., Victoria: Trafford Publishing, 2007.

[8] B. H. Cumpston, et al:: Two-photon polymerization initiators for three-dimensional optical data storage and microfabrication, Nature, vol. 398, pp.51-54, 1999.

[9] S. Kawata, et al.: Finer features for functional microdevices", Nature, vol. 412, pp.697-698, 2001.

[10] J. Li, et al.: F2-laser digital etching of colloidal photonic crystals, Appl. Phys. Lett., vol.87, 141106, 2005.

[11] W. Lee, et al: : Multiphoton polymerization of waveguide structures within three-dimensional photonic crystals, Adv. Mater., vol. 14, pp.271-274, 2002.

[12] S. Pruzinsky, P. Braun, Fabrication and characterization of two-photon polymerized features in colloidal crystals, Adv. Funct. Mater., vol.15, pp.1995-2004, 2005. 